\title{
Improving theatre efficiency and utilisation through early identification of trauma patients and enhanced communication between teams
}

\author{
Simon Roberts, Adnan Saithna, Rob Bethune \\ George Eliot Hospital
}

\begin{abstract}
Surgical departments are increasingly put under pressure to improve services, cut waiting lists, increase efficiency and save money. At a district general hospital in the west-midlands we approached the challenge of improving efficiency and optimising the services available in our orthopaedic theatres.

Data was collected on: anaesthetic start times, operation start and finish times, and reasons for delay in our trauma theatre over a period from October 2014 to January 2015. During this period a change was implemented to improve the start time of the first operation of each day in the trauma theatre. Through adaptation of a method developed by Javed $S$ et al, a patient was pre-selected by the on-call team and given the name the "golden patient" the day before they were due to be operated upon. This nominated patient would then be fixed at the start of the trauma theatre list the following day. The list would only then change if a "life or limb threatening" case was admitted overnight. The on-call team would prioritise that this patient was optimised for theatre and the theatre staff would ensure the surgical instruments were prepared.
\end{abstract}

A PDSA cycle method was used, collecting data on 80 orthopaedic trauma cases during the period, and demonstrated a 59 minute $(95 \% \mathrm{Cl}$ 45-72) improvement in start times from 10:49 AM to 9:50 AM with a p-value of 0.00024 with the intervention of early allocation of the first patient on the trauma list.

A relatively simple intervention tool designed to improve communication within and between health-care teams can have a significant impact on the efficiency of a complex environment such as a trauma theatre.

\section{Problem}

At The George Eliot Hospital (Nuneaton, UK), theatre utilisation in orthopaedics has been identified as suboptimal on the basis of previous audit. This data demonstrates that the first case can often begin at 10:30 AM when the intended start time is 9:00 AM. In addition, there can be significant delays between each case.

The trauma list is affected most significantly and can be delayed up to two hours between cases. From discussion with various members of the theatre team it seems that the reasons for the delays are multifactorial but are often considered to be predictable and preventable. Examples include the patient not having been sent for, the patient's being fed, outstanding investigations (bloods, ECG, echocardiogram etc), unavailable equipment, and inadequate staffing levels.

The delays between cases typically means that the operating lists overrun and this leads to further delays for pending cases. Additional contributing factors include inadequate staffing levels and other demands on the emergency theatre (for example general surgical emergencies which share the list and often take priority). Furthermore, if an elective list overruns, evening staff are taken from the emergency list resulting in an inability to perform work in the emergency theatre creating further delays and cancellations.

The cancellations and delays can in theory compromise the quality of care of those patients awaiting surgery. They undermine the timeliness, efficiency and effectiveness of care, and they are often not patient-centred. They also have secondary effects. Delays may lead to increased length of stay, increasing hospital acquired infections, and creating significant dissatisfaction for patients and relatives. They increase both the bed days of the patient and hours the operating theatre is not in use; this creates considerable cost to the trust.

\section{Background}

George Eliot hospital has two elective operating theatres for eight orthopaedic consultants. As well as this, there is an emergency list which dedicates at least one session to orthopaedics each day. Further sessions are offered to obstetrics and gynecology and general surgery.

Just like in any other hospital, the theatres in George Eliot work like complex systems. They require a multitude of healthcare professionals to work in harmony for it to function. Each team member must provide their skill, in a time crucial manner, for the theatre to operate efficiently.

The operating room is home to both life saving, and quality of life saving intervention. It is also one of the most expensive things to maintain for an NHS trust. Therefore, effective cohesion, utilisation, and efficiency of: the theatre space, preoperative planning, and 
theatre staff are of paramount importance when considering improvements to that system.

Delays to operations are often multi-causal and can involve any step of the patient journey.

A Medline search of recent literature investigating theatre efficiency and delays was conducted in order to provide an evidence-base to guide us in this service improvement project. Javed, et al. demonstrated improved start times in their trauma theatre through preselection of an investigated, prepared patient awaiting trauma surgery. This study was performed in the Royal Preston Hospital in 2013; a major trauma centre for the local area. They named the prechosen patient the "golden patient", and failing a life or limb threatening patient being admitted this patient was "locked in" to be first on the trauma-list the following day. Through this method, they demonstrated a 30 minute improvement in theatre start time from 10:03 AM to 9:33 AM.[1]

In a Canadian prospective observational study from 2009, Wong, et al. measured delays of 1500 patients attending surgery over a nine year period and found that the majority of delays are hospital-wide factors. These are made up from lack of ward beds, slow transfers, and patients not ready on wards. This was followed by doctor factors: consent not completed, surgeon delayed, or medication not prescribed. Other factors relate to theatre kit preparations, lack of nursing or recovery staff, and IT failure. One notable extraction from the review seems to be that delay of the first case of the day correlates significantly with further delays in the day.[2]

Ciechanowicz, et al. performed a prospective survey recording starttime and total daily delay over five days in March 2010 in a district general hospital. They collected data on 200 cases recording reasons for delays along with a cost analysis for the trust based on lost theatre hours. Overall $78 \%$ of cases started on time.

Orthopaedics achieved only $69 \%$, exceeding the acceptable total daily delay time of 45 minutes. Hospital-wide factors were the most common reason for the delays ( $72 \%)$. Forty-eight percent were due to ward bed issues, $15 \%$ due to "doctor" factors, and $13 \%$ to inadequate pre-operative assessment. The estimated projected cost to the Trust over one year was significant at over £1 million (\$1.5 million).[3]

Pandit, et al. demonstrated a significant (6\%-60\%) improvement in start-time with the use of a theatre facilitator on an orthopaedic trauma list.[4] Christopher, et al. aimed to define theatre efficiency and did so as "maximising utilisation, minimising over-running and minimising cancellations on a list." Through models they demonstrated that incorporations all of these aspects of efficiency can yield the most effective improvements in theatre efficiency.[5]

\section{Baseline measurement}

Data was collected over a three week period in a prospective manner from both the elective and trauma orthopaedic theatres. We prioritised the accurate collection of the timings of each step of the surgery. These included the times: sent for, arrival in anaesthetic room, arrival in theatre, start of operation, end of operation, out of theatre and time the next patient was sent for. Each case used its own data collection form and the data collector was present at each step of the data collection point to ensure accurate readings.

If at any point there was a delay in any parameter of this process, this was documented and reasons/opinions from each health-care discipline was sort: surgeon, anaesthetist, scrub nurse, ODP, theatre staff. The data collection forms provided all sourced reasons for delay as well as those obtained from literature and allowed a tickbox style collection to be made. There was also a free text box for any reasons for delay which did not fit into the printed list. The reasons for delay were collected along with opinions on how the problem could have been avoided or how the situation leading to the delay could have been improved. As well as this, we made note of whether a consultant or registrar was operating when the teambrief was performed and if any issues were highlighted during the team brief.

We collated the data and categorised the causes of the delays and timings, noting the team members identifying the problems as well as possible improvements. We collated the mean times of each of the parameters collected such as the time patients were called for and the time of start of the operation. Furthermore, in those incidences with delays, we numbered and ranked the reasons for delays and examined these along with the changes that were proposed.

See supplementary file: ds4547.docx - "Quality Improvement Project - data collection sheet"

\section{Design}

The first PDSA cycle of the audit was presented to the hospital directorate. This included members of the orthopaedic, anaesthetic, theatre and financial teams. The problems identified in the study were discussed alongside potential solutions of improving efficiency.

The first cycle highlighted that significant issues arose from delays in the trauma theatre, which we subsequently prioritised as an area to improve efficiency.

A large prospective study by Javed et al performed in Preston suggested that identifying a specific patient for the first case of the day (dubbed the "golden patient"[1]) would improve the start times of the first case in the trauma theatre.

Early identification of the first patient to be operated upon the following day in the trauma theatre was chosen to be our main intervention. We decided the on-call registrar, in communication with the consultant, would determine the golden patient. This patient would then be fixed as first on the list the following day, unless a life or limb threatening case was admitted overnight. It would then be the responsibility of the on-call team to disseminate the information to all parties involved in the patient's care. The medical team could then focus on ensuring the patient was optimised for theatre and the ward could ensure the necessary checks were performed in good time. The theatre team would then 
have enough time to prepare the equipment for the case.

A nominated member of each the team would have the responsibility of ensuring all conceivable problems could be identified and addressed well in time for surgery.

These are the responsibilities of the relevant staff involved. Firstly, it is the responsibility of the night on-call orthopaedic SHO to ensure that the patient is: consented, marked, investigations are completed, reviewed and actioned, the ward are aware of NBM status, IV fluids are prescribed, VTE prophylaxis is prescribed and the theatres are informed of the patient and the operation. As well as this, they must update the trauma board. The anaesthetic team will hold the responsibility of reviewing the patient, ensuring that all investigation they need ahead of anaesthetic have been performed and voicing any concerns they hold to the orthopaedic team. The ward staff nursing the patient hold the responsibility of performing their ward-checks and ensuring the orthopaedics and anaesthetic team have performed their roles. A "golden patient" form will sit in the front of the notes and hold a check list for each of the teams to sign when their duty has been completed. This will also allow theatres ample time to ensure that their equipment is complete and ready for the first case.

Our second intervention, to again address communication issues, was to set up a live trauma board. This will be a virtual trauma board which contains the pertinent information of each patient that requires emergency orthopaedic surgery. The information held will include: date of admission, patient details, location, consultant incharge, injury, operation proposed, date for surgery, whether a specific surgeon is required, order on list and if it is a fractured neck of femur, the time of expiry of the 36 hour deadline. This form will be updated throughout the day with the new admissions and removing those patients whose operations have been performed.

We further discussed and considered other interventions: ring fence beds for orthopaedic patients, a trauma facilitator, staggering recovery nurses start times, and more recovery beds. Consensus was made that these can be considered following the review of the current interventions.

\section{Strategy}

PDSA cycle 1: The golden patient forms were added to the orthopaedic trauma ward and into the orthopaedic folder on the intranet, which is accessible anywhere in the trust. The orthopaedic team were instructed that a nominated patient needs to be identified each day and this must be confirmed by the 8:00 PM handover at the latest each day.

The initial week of installation of the new change was successful. The project team were on-call at night and able to initiate the changes as well as this we were able to work as a proxy theatrefacilitator. This ensured all components of the golden patient form had been completed and the patient had been discussed with the appropriate team members to allow the patient to be ready to be sent for by 8:00 AM. Over the following two weeks, the on-call night $\mathrm{SHO}$ was effectively briefed prior to their week of nights, discussions took place during the morning identifying pitfalls involving the on-call registrar and consultant. This again proved to be successful maintaining the improved start times.

The following weeks were significantly less impactful. The golden patient was not consistently used by the team each day and a patient was chosen only if the team remembered to do so. As such the project demonstrated a far less dramatic change in start times. The trend seemed to suggest that without active intervention from the project team the changes would not be sustained and the start times would return back to baseline.

PDSA cycle 2: In order to address this issue we re-implemented the changes and discussed with the teams how we could ensure that the intervention is used. The main obstacle proved to be that on busy shifts the golden patient idea was either forgotten or not considered a priority in terms of time management. We therefore made it a mandatory part of the evening nursing handover that the nominated golden patient was identified. If one had not already been identified the on-call SHO could be reminded. This produced similar results to the first weeks of the study.

PDSA cycle 3: In the final weeks of the trial we took a less active role in theatre facilitation as well as reminding the team to choose a patient, allowing us to test the sustainability of this model. The resulting weeks demonstrated a consistent use of the intervention this demonstrated a consistent improvement in theatre start times.

See supplementary file: ds4648.docx - "PDSA Cycles (1)"

\section{Post-measurement}

The data collection was performed over a twelve week period from October 2014 to January 2015. Six weeks of which, were prior to the intervention and six weeks of which were after the introduction of the "golden patient".

The attached charts shows a running plot of the aggregated data of the 12 weeks, split into 16 data points; with the intervention taking place on point 9 .

Prior to the intervention, the mean time the first patient was sent for was 8:48 AM. The mean time in the anaesthetic room was 9:28 AM and the mean operation start time was 10:49 AM (Cl 10:43 AM to 10:55 AM).

After the golden patient intervention, the start time decreased to an average of 9:50 AM (CI 9:36 AM to 10:03 AM) over the six week period. This demonstrated a 59 minute improvement in mean start times ( $p$-value $=0.00024)$.

The charts attached, display data points in minutes after 9:00 AM. They show a significant improvement in start times following the intervention. All data points are lower than the pre-intervention mean. This is indicative of special cause variation, the conclusion of which would suggest that the use of the golden patient model has a causal relationship to the improvement in operation start-times in the trauma theatre. 
See supplementary file: ds4750.pptx - "Charts of theatre start times"

\section{Lessons and limitations}

The initial aim of the study was to improve the efficiency of the orthopaedic theatres at George Eliot Hospital. We planned to achieve this by starting the cases earlier and cutting wasted time though optimising the facilities available but this was far too ambitious. This was a broad aim attempting to address too many variables. The preliminary data collection was very useful in narrowing our objectives, it provided the opportunity to identify a single significant problem that needed to be addressed as well as highlighting strategies for overcoming the problem.

The project provided many opportunities for learning: time management and organisation, communication skills, IT and statistical skills, management and leadership skills.

\section{Conclusion}

A relatively simple intervention tool designed to improve communication within and between health-care teams can have a significant impact on the services provided to patients. The golden patient form provided an easy to use checklist reminder for each professional body to allow an efficient service to be provided with minimal external input from senior clinicians and managers.

The impact of the tool can improve the care delivered to trauma patients in Nuneaton and potentially pave the wave for further improvements in the trust. As NHS trusts are continuously put under greater demands to improve efficiency of service without increasing costs this could potentially represent a small victory in the fight to improving the NHS.

\section{References}

1. Javed S, Peck C, Salthouse D, Woodruff MJ. A predetermined first patient on the trauma list can improve theatre start times. Injury 2013;44(11):1528-31.

2. Janice Wong, Kathleen Joy Khu, Zul Kaderali, Mark Bernstein. Delays in the operating room: signs of an imperfect system. Can J Surg 2010 June; 53(3):189-95.

3. S Ciechanowicz, N Wilson. Delays to Operating Theatre Lists: Observations from a UK Centre. Internet $\mathrm{J}$ Health 2010;13(1)

4. Panni MK, Shah SJ, Chavarro C, Rawl M, Wojnarwsky PK, Panni JK. Improving operating room first start efficiency value of both checklist and a pre-operative facilitator. Acta Anaesthesiol Scand 2013 Oct;57(9):1118-23.

5. Pandit JJ, Westbury S, Pandit M. The concept of surgical operating list 'efficiency': a formula to describe the term. Anaesthesia 2007 Sep;62(9):895-903.

6. Christopher L. Delaney, Nathan Davis, Peter Tamblyn. Audit of the utilization of time in an orthopaedic trauma theatre. ANZ J Surg 2010 April; 80(4):217-22. doi:

10.1111/j.1445-2197.2009.05043.x.

7. D. Ricketts, J. Hartley, M. Patterson, W. Harries, and D.
Hitchin. An orthopaedic theatre timings survey. Ann R Coll Surg Engl May 1994; 76(3):200-4.

8. James G. Wright, Ann Roche, Antoine E. Khoury. Improving on-time surgical starts in an operating room. Can J Surg 2010 June; 53(3):167-70.

\section{Declaration of interests}

None.

\section{Acknowledgements}

Mr George Joseph, Dr Phillip Harrington. 